\title{
Survey of echinococcoses in southeastern Qinghai Province, China, and serodiagnostic insights of recombinant Echinococcus granulosus antigen $B$ isoforms
}

Xiumin Han ${ }^{1,2+}$, Jeong-Geun Kim ${ }^{3 \dagger}$, Hu Wang ${ }^{2,4}$, Huixia Cai ${ }^{2,3}$, Xiao Ma ${ }^{2}$, Duc Hieu Duong ${ }^{3}$, Chun-Seob Ahn ${ }^{3}$, Insug Kang ${ }^{5}$ and Yoon Kong ${ }^{3 *}$

\begin{abstract}
Background: Echinococcoses, caused by metacestodes of Echinococcus granulosus (cystic echinococcosis; CE) and E. multilocularis (alveolar echinococcosis; AE), represent major emerging parasitic diseases. These enzootic helminthiases invoke significant public health concerns and social burdens in endemic areas. The diseases are prevalent in the Qinghai-Tibetan Plateau, China, while community-based epidemiological studies have been scarcely reported. We surveyed echinococcosis patients in the southeastern Qinghai Province, China, to better understand the concurrent epidemiological situation in this area.
\end{abstract}

Methods: During July and August of 2013 and 2014, we screened echinococcosis patients at Yushu and Golog Prefectures, Qinghai Province, China, in a diagnostic campaign. A total of 2856 people (male:female ratio, 1:1.12; mean age, 34.6 years; age range, 6-88 years) were ultrasonographically examined for the presence of hepatic echinococcal cysts. We also collected serum samples from patients and analyzed antibody reactivity against recombinant forms of diverse E. granulosus antigen Bs (rEgAgB1-5) by enzyme-linked immunosorbent assay.

Results: We detected 134 patients whose imaging scans were compatible with CE (115 cases) and AE (20 patients). One patient might have been infected with both $C E$ and $A E$. The overall incidence was 4.7\% (CE, 4.0\%; $A E, 0.7 \%)$. A large proportion (67.5\%) of CE patients was diagnosed at active and transitional CE1-CE3 stages in their late 30s. The AE cases were generally detected at advanced stage in patients at early 20s (60\%). Analysis of the receiver operating characteristic curve and Youden's index indicated that rEgAgB2 was the most promising biomarker, followed by rEgAgB3 and rEgAgB1. Overall, sensitivity and specificity of rEgAgB1-3 were 84.5-92.7\% and 91.9-94.6\%, respectively. rEgAgB4 and 5 showed low sensitivity with high cross-reactivity.

Conclusions: Our results strongly suggest that disability-adjusted life years related to echinococcoses in QinghaiTibetan areas might be more serious than previously considered. Control and prevention strategy against CE and AE are highly required in these areas. In addition to ultrasonography, serological tests might provide supportive data. However, serological data should be carefully interpreted for differential diagnosis, especially in areas where both CE and $\mathrm{AE}$ are co-endemic.

Keywords: Echinococcoses, Echinococcus granulosus, E. multilocularis, Qinghai-Tibetan Plateau, Ultrasonography, Antigen B isoforms, Immunoreactivity

\footnotetext{
*Correspondence: kongy@skku.edu

${ }^{\dagger}$ Xiumin Han and Jeong-Geun Kim contributed equally to this work

${ }^{3}$ Department of Molecular Parasitology, Samsung Medical Center,

Sungkyunkwan University School of Medicine, Suwon 16419, Korea

Full list of author information is available at the end of the article
} 


\section{Background}

A number of Echinococcus spp. cause echinococcoses during their metacestode stage, among which Echinococcus granulosus and E. multilocularis invoke major human diseases, cystic echinococcosis (CE) and alveolar echinococcosis (AE). Humans serve as an accidental intermediate host and are infected with these parasites by contracting eggs expelled from canine definitive hosts. Ingested eggs are activated in the small intestine, released into the bloodstream, and primarily end up lodged in the liver. Echinococcus granulosus metacestode typically forms a unilocular cyst that induces a space-occupying lesion whereas $E$. multilocularis metacestode progresses to a multivesiculated cystic mass that infiltrates into the nearby hepatic parenchyme with behavior resembling invasive malignant tumors. AE sometimes establishes metastatic lesion(s) in other remote tissues/organs [1].

Echinococcoses represent major emerging enzootic parasitic diseases, which have resulted in significant medical problems and public health concerns in endemic areas. CE is prevalent in nomadic areas of Europe, Central and Middle Asia, Africa, Australia, South America and northwestern China in association with dog-rearing environments [1]. Approximately four million people are infected and another 40 million people are at risk annually $[2,3]$. AE is endemic to several communities in Eurasia, North America and northwestern China. The overall annual incidence of AE has been reported to be $0.2-6.0$ per 100,000 inhabitants $[4,5]$. AE exemplifies one of the most fatal helminthic diseases. Its lethality has been reported to be substantially increased if not treated properly [6]. In recent years, patients infected with E. granulosus and E. multilocularis have been increasingly detected in northern parts of China, including Xinjiang, QinghaiTibetan Plateau and Heilongjiang areas. In these areas, the seroprevalence rate of CE is approximately $5-30 \%$ of the inhabitants and that of $\mathrm{AE}$ is as high as $6 \%$ in some village populations $[7,8]$.

The diagnosis of echinococcoses largely depends on imaging scans and serological tests. Demonstration of typical lesions by ultrasonographic (US) examination has been the diagnosis of choice. US diagnosis has widely been used in both field survey and bedside diagnosis. The procedure is beneficial because it is easy to apply and is not invasive. Moreover, it has the definitive merit of providing an immediate diagnosis [9-12]. However, differential diagnosis of echinococcoses from hepatic cyst(s)/ mass(es) with other etiologies such as hemorrhagic cyst, pyogenic/infectious abscess, cystadenoma, liver cirrhosis, primary hepatocellular carcinoma and metastatic tumors is often required and sometimes difficult, even for experienced physicians [13].
Serological tests might compensate for this shortcoming and provide additional evidence for echinococcoses [14-16]. Several antigenic proteins have been characterized to analyze their serodiagnostic performance, including E. granulosus antigen 5 (EgAg5), diverse antigen B subunits (EgAgBs) and endophilin B1. These molecules show sensitive antibody responses against patient sera from CE cases. However, the diagnostic applicability of EgAg5 and endophilin B1 is compromised by the lack of specificity $[17,18]$. EgAgB proteins are abundantly found in hydatid fluid (HF) and are shown to induce strong antibody responses to sera from infected individuals [1416]. Echinococcus multilocularis metacestodes develop into multivesiculated masses containing minute vesicular fluid. However, E. multilocularis antigen B proteins (EmAgBs), which are orthologs of EgAgBs, are also found in E. multilocularis HF [19]. EmAgB3 is abundantly expressed in the early developmental stage and exhibits specific antibody responses against sera from patients with early stage $\mathrm{AE}[19,20]$.

Five distinct EgAgB isoforms (EgAgB1-5) have been synthesized from at least ten unique genes [21], among which EgAgB1 and EgAgB2 molecules have been reported to be reliable serodiagnostic biomarkers for $\mathrm{CE}$ [22-25]. However, conflicting data have been repeatedly observed depending on experimental conditions [22, 24, 25]. More importantly, there have been a limited number of reports regarding serological cross reactivity of $\mathrm{AE}$ patient sera against EgAgB molecules $[15,26]$. The diagnostic reliability of EgAgBs should be re-evaluated for their actual feasibility.

In the present study, we surveyed echinococcosis patients in the Qinghai-Tibetan Plateau to better understand the epidemiological situation in this area. We diagnosed patients by abdominal US examination and collected blood samples. We subsequently analyzed differential immunoreactivity segregated to distinct EgAgB isoforms to determine their diagnostic capability.

\section{Methods}

Patient survey and collection of serum samples

During July and August of 2013 and 2014, we surveyed echinococcosis patients at Chengduo and Yushu Counties (Yushu Tibetan Autonomous Prefecture) and Dari and Banma Counties (Golog Tibetan Autonomous Prefecture), Qinghai Province, China, in a health promotion campaign. These places were highland seminomadic areas approximately 600-900 km away from Xining (Fig. 1). The study purpose was explained to local health authorities, school teachers, community leaders and residents to ensure their acceptance and participation. We screened 2856 people (male:female ratio, 1:1.12; mean age, 34.6 years; age range, $6-88$ years) by abdominal US 


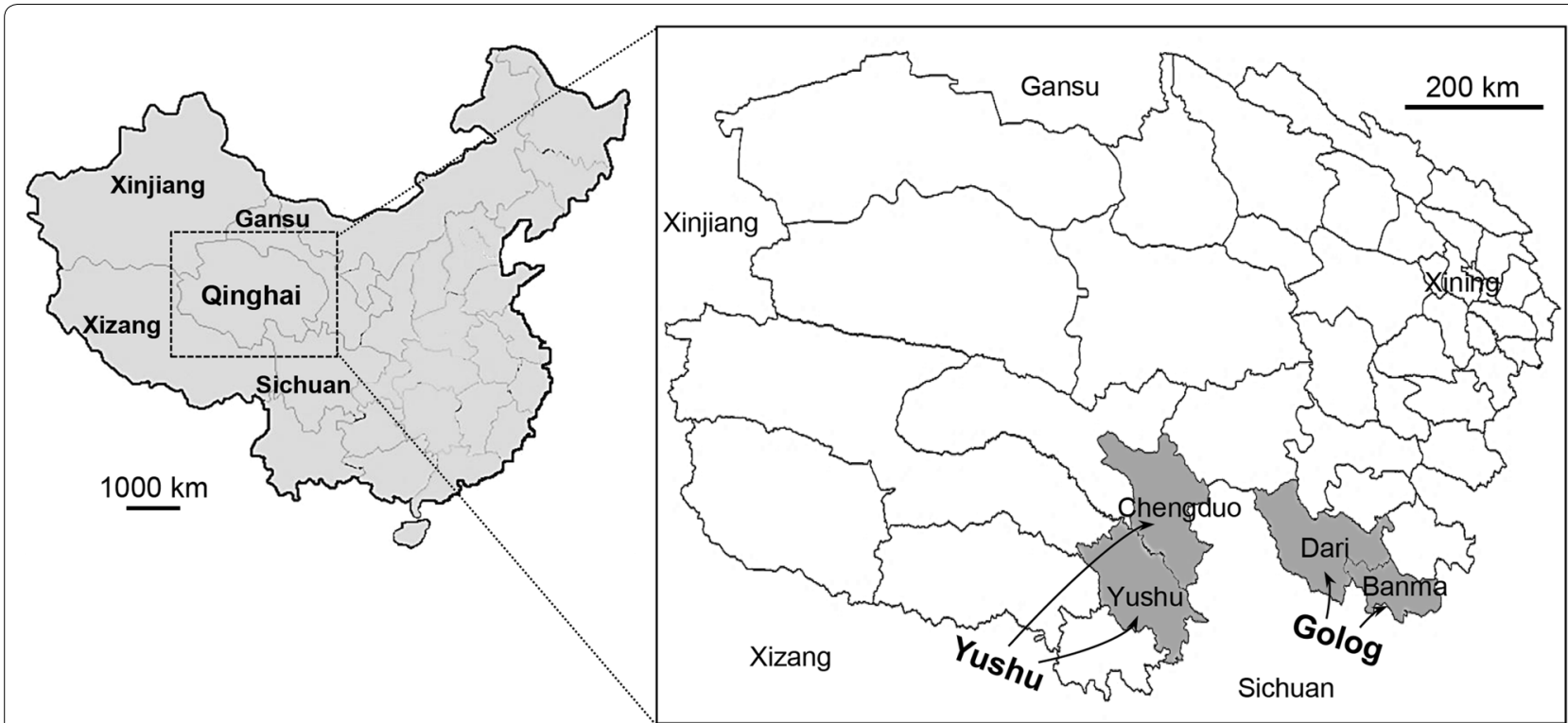

Fig. 1 Map showing surveyed areas. We surveyed patients as part of diagnostic campaigns for echinococcoses at Chengduo and Yushu Counties (Yushu Tibetan Autonomous Prefecture) and Dari and Banma Counties (Golog Tibetan Autonomous Prefecture), Qinghai Province, China, during July and August of 2013 and 2014

Table 1 Age and sex distribution of persons surveyed and patients identified based on abdominal ultrasonography

\begin{tabular}{|c|c|c|c|c|c|c|c|c|c|}
\hline \multirow[t]{2}{*}{ Age (years) } & \multicolumn{3}{|c|}{ No. examined (\%) } & \multicolumn{3}{|c|}{ No. positive (\%) } & \multicolumn{3}{|c|}{ No. of healthy controls (\%) } \\
\hline & Male & Female & Total & Male & Female & Total & Male & Female & Total \\
\hline$<10$ & 175 & 182 & 357 & $4(2.3)$ & $4(2.2)$ & $8(2.2)$ & $1(0.6)$ & $2(1.1)$ & $3(0.8)$ \\
\hline $10-19$ & 358 & 428 & 786 & $14(3.9)$ & $18(4.2)$ & $32(4.1)$ & $6(1.7)$ & $7(1.6)$ & $13(1.7)$ \\
\hline $20-29$ & 201 & 245 & 446 & $7(3.5)$ & $6(2.5)$ & $13(2.9)$ & $3(1.5)$ & $3(1.2)$ & $6(1.3)$ \\
\hline $30-39$ & 289 & 302 & 591 & $10(3.5)$ & $16(5.3)$ & $26(4.4)$ & $5(1.7)$ & $7(2.3)$ & $12(2.0)$ \\
\hline $40-49$ & 151 & 161 & 312 & $13(8.6)$ & $15(9.3)$ & $28(9.0)$ & $7(4.6)$ & $7(4.3)$ & $14(4.5)$ \\
\hline $50-59$ & 95 & 104 & 199 & $4(4.2)$ & $7(6.7)$ & $11(5.5)$ & $2(2.1)$ & $4(3.8)$ & $6(3.0)$ \\
\hline$>60$ & 77 & 88 & 165 & $10(13.0)$ & $6(6.8)$ & $16(9.7)$ & $5(6.5)$ & $3(3.4)$ & $8(4.8)$ \\
\hline Total & $1346(47.1)$ & 1510 (52.9) & $2856(100)$ & $62(4.6)$ & $72(4.8)$ & $134(4.7)$ & $29(2.2)$ & $33(2.2)$ & $62(2.2)$ \\
\hline
\end{tabular}

examination (Aloka SSD 500, Ganim Medical, Delaware, $\mathrm{OH}$, USA). In patients with hepatic space-occupying lesion(s) compatible with echinococcoses (Additional file 1: Figure S1), power Doppler sonography was additionally conducted to observe the distribution of blood flow. Blood samples were collected from persons who understood our purpose and agreed to participate. We were able to collect blood samples from 122 patients. In addition, we collected blood samples randomly from 62 age- and sex-matched apparently healthy persons (male:female ratio, 1:1.14; mean age, 31.4 years; age range, 9-67 years) who did not show any abnormality on abdominal US (Table 1). They denied possible exposure to parasitic infections including protozoans. All patients and healthy donors enrolled in this study were dog owners from the same villages with similar occupations (semi-nomads and semi-farmers). Patients who had already been diagnosed as echinococcoses were excluded from statistical analyses $[27,28]$.

\section{Cloning and expression of recombinant antigen B subunits (rEgAgBs)}

mRNA sequences of EgAgBs (B1, EgrG_000381200; B2, EgrG_000381100; B3, EgrG_000381600; B4, EgrG_000381400; and B5, EgrG_000381800) were retrieved from GeneDB (http://www.genedb.org/Homep age/Egranulosus). Total RNA was extracted from a single ovine CE2 cyst using an RNA extraction kit (iNtRON, Seongnam, Korea). cDNA was reverse transcribed using a first strand cDNA synthesis kit (Invitrogen, Carlsbad, 
CA, USA). Mature domains of respective genes were amplified with gene-specific primers (Additional file 2: Table S1). PCR cycling conditions were as follows: denaturation at $95{ }^{\circ} \mathrm{C}$ for $5 \mathrm{~min}, 35$ cycles of $95^{\circ} \mathrm{C}$ for $1 \mathrm{~min}$, $50{ }^{\circ} \mathrm{C}$ for $45 \mathrm{~s}$ and $72{ }^{\circ} \mathrm{C}$ for $1 \mathrm{~min}$, with a final extension step at $72{ }^{\circ} \mathrm{C}$ for $5 \mathrm{~min}$. Each plasmid was digested with restriction enzymes, ligated into a pGEX-6p-1 expression vector (Novagen, Cambridge, MA, USA) and transformed into Escherichia coli BL21 (DE3) competent cells. Recombinant proteins fused with glutathione transferase (GST) were expressed by treatment with $1 \mathrm{mM}$ isopropyl $\beta$-D-1-thiogalactopyranoside (IPTG) at $37{ }^{\circ} \mathrm{C}$ for $4 \mathrm{~h}$. Recombinant proteins were purified using glutathioneSepharose 4B beads (GE Healthcare, Piscataway, NJ, USA), after which the GST-tag was removed by PreScission protease (GE Healthcare). Homogeneity of purified proteins was monitored by Tricine SDS-PAGE (10\%) with Coomassie brilliant blue R-250 (CBB) staining.

\section{Enzyme-linked immunosorbent assay (ELISA)}

After a checkerboard titration, $100 \mu \mathrm{l}$ of each rEgAgB $(1.5 \mu \mathrm{g} / \mathrm{ml}$ suspended in $100 \mathrm{mM}$ carbonate-bicarbonate buffer, pH 9.6) was used to coat the wells of a flat-bottom 96-well microplate (Greiner Bio-One, Kremsmuenster, Austria) overnight at $4{ }^{\circ} \mathrm{C}$. One hundred microliters of serum samples diluted 1:100 in phosphate buffered saline containing $0.05 \%$ Tween 20 (PBS/T) were incubated at $37{ }^{\circ} \mathrm{C}$ for $2 \mathrm{~h}$, after which $100 \mu \mathrm{l}$ of horseradish peroxidase-conjugated anti-human IgG antibody (1:4000 dilution in PBS/T; MP Biochemicals, Santa Ana, CA, USA) was further incubated at $37{ }^{\circ} \mathrm{C}$ for $2 \mathrm{~h}$. Color reactions were developed with $100 \mu \mathrm{l}$ of $1 \% o$-phenylenediamine (Sigma-Aldrich, St. Louis, MO, USA) supplemented with $0.03 \% \mathrm{H}_{2} \mathrm{O}_{2}$ for $20 \mathrm{~min}$ in the dark. Reactions were halted by adding $50 \mu \mathrm{l}$ of $2 \mathrm{~N} \mathrm{H}_{2} \mathrm{SO}_{4}$ (Sigma-Aldrich). The absorbance was measured at $450 \mathrm{~nm}$ on an NEO microplate reader (Biotek, Winooski, VT, USA). Sera from healthy donors and PBS/T were used as negative and blank controls, respectively. All results were determined after correction with appropriate blank. Each sample was independently assayed in triplicate.

\section{Statistical analyses}

We used positive/negative $(\mathrm{P} / \mathrm{N})$ values to normalize data from different batches of ELISA results. Receiver operating characteristic (ROC) curves were generated by plotting sensitivity (\%) versus 100 -specificity (\%). The area under the ROC curve (AUC) was employed to conduct pairwise comparisons of diagnostic performance. We determined cut-off values using ROC analyses and defined them as $\mathrm{P} / \mathrm{N}$ values that rendered the highest sum of sensitivity and specificity. Graphical representations were created employing GraphPad Prism v.7.0.
Accuracies of diagnostic tests such as sensitivity, specificity, Youden's index, positive/negative likelihood ratios (PLR/NLR), and positive/negative predictive values (PPV/NPV) were determined using MedCal (free statistical calculators and diagnostic test evaluation calculator) with 95\% confidence interval (CI). Student's t-tests and one-way analysis of variance (ANOVA) were used to compare groups. Significance was set at $P<0.05$.

\section{Results}

\section{Ultrasonographic diagnosis of CE and AE patients}

We surveyed echinococcosis patients at two counties each in Yushu (Yushu and Chenduo Counties) and Golog (Dari and Banma Counties), Tibetan Autonomous Prefectures (Fig. 1). A total of 2856 people were examined by abdominal US for the presence of hepatic echinococcal cysts. People aged between 10 and 40, those who were physically healthy, and with strong socioeconomic activities comprised the majority $(74.8 \%)$ of the study population. Most men were half-nomadic and half-farmers while most women were housewives. The young age group less than 10 years-old constituted $12.5 \%$ of participants and those who were older than 50 years accounted for $12.7 \%$ of participants. No difference between males and females was observed in these subjects (male:female $=1: 1.12$; $t_{(12)}=0.3948, P=0.6999$ ) (Table 1 ).

We were able to detect 134 new patients whose imaging scans were compatible with $\mathrm{CE}$ or $\mathrm{AE}$, among which 115 cases showed US images probably representative of CE and 20 cases of AE [20, 29-31] (Table 2). Gender difference was not observed for disease incidence $\left(t_{(12)}=0.1622, \quad P=0.8733\right)$. A 48-year-old herdsman at Dari County (Golog Prefecture) was found to be

Table 2 Patients with cystic echinococcosis (CE) and alveolar echinococcosis (AE) diagnosed by ultrasonographic findings

\begin{tabular}{lllll}
\hline Disease category & Stage & \multicolumn{3}{l}{ No. of patients (mean age, years) } \\
\cline { 3 - 5 } & & Male & Female & Total $^{\mathrm{a}}$ \\
\hline CE & CE1 & $17(33.2)$ & $10(20.3)$ & $27(28.4)$ \\
& CE2 & $6(41.2)$ & $13(31.3)$ & $19(34.4)$ \\
& CE3a & $5(33.6)$ & $5(34.8)$ & $10(34.2)$ \\
& CE3b & $5(25.4)$ & $16(39.4)$ & $21(35.9)$ \\
& CE4 & $9(43.6)$ & $14(35.9)$ & $23(38.7)$ \\
Subtotal & CE5 & $10(52.3)$ & $5(46.0)$ & $15(50.2)$ \\
AE & & $52(38.9)$ & $63(34.1)$ & $115(36.2)$ \\
& Early AE & $5(18.6)$ & $3(27.3)$ & $8(21.9)$ \\
Subtotal & Advanced AE & $6(24.8)$ & $6(21.3)$ & $12(23.1)$ \\
Total & & $11(22.0)$ & $9(23.3)$ & $20(22.6)$ \\
\hline
\end{tabular}

a Total number exceeded 134 due to one case with double infection of both $\mathrm{AE}$ and $C E$ 
concomitantly infected with a CE5 cyst and an early AE mass (Fig. 2), although we could not confirm his diagnosis by histopathological examination.

Representative ultrasonograms of CE patients are shown in Additional file 1: Figure S1a. Most CE1 patients revealed typical US findings of well-defined round unilocular hypodense cystic mass(es) with variable sizes (3.1-8.5 cm in diameter). Snow-flake sign and/or doubleline sign were typically observed. CE2 patients exhibited multiseptated daughter cysts within a unilocular large mother cyst with size ranging between $7.8-11.1 \mathrm{~cm}$ in diameter. CE3a cysts showed a detachment of laminated endocyst (water-lily sign) and CE3b cysts contained solid matrix with daughter cysts (wheel-like appearance). Cyst sizes were variable $(6.4-9.5 \mathrm{~cm})$. CE4 patients demonstrated heterogeneous hypoechoic degenerative contents. Degenerating membranes revealed canalicular structure (ball of wool appearance). Cyst sizes varied between 6.3$8.8 \mathrm{~cm}$. CE5 stage was characterized by a highly degenerative cyst with thick calcified wall. Of $115 \mathrm{CE}$ patients, transitional CE3 stage was detected the most frequently (27\%, 31/115 cases; 10 and 21 patients with CE3a and CE3b, respectively), followed by CE1 $(n=27)$, CE4 $(n=23)$, CE2 $(n=19)$ and CE5 stages $(n=15)$.

Active CE1 and CE2 cases were initially diagnosed at a mean age of 31.6 years $(n=46)$ while those with transitional CE3a/b stages were 34.1 years (CE3a) and 35.6 years (CE3b), respectively. Chronic inactive CE4 and CE5 stages were generally detected in patients in their late $30 \mathrm{~s}$ to 50 s (mean age of 38.7 and 50.8 years for CE4 and CE5, respectively, Table 2). Involvement of the right hepatic lobe was commonly observed (70.4\%, 81/115 cases). Multiple cysts were recognized in $16(13.9 \%)$ patients.

Among 20 cases of $\mathrm{AE}$, eight patients were suspected to be in the early stage because their imaging scans disclosed a mass containing multiple echogenic small nodules (hailstorm appearance) with indistinct borders combined with or without scattered calcifications in the mass. Another 12 patients showed US findings consisting of a central hypoechogenic mass combined with peripheral hyperechoic indistinct and irregular margins that were compatible with advanced stage $\mathrm{AE}$ (Table 2). Sizes of these masses were variable $(3.4-7.8 \mathrm{~cm}$ in diameter). Typical US features are presented in Additional file 1: Figure S1b. No significant difference in age at the time of diagnosis was observed between early and advanced stages (mean age: 22.3 years versus 23.1 years; $\left.t_{(18)}=0.8285, P=0.4183\right)$. All patients had a single lesion predominantly in the right lobe $(80 \%, 16 / 20$ cases).

Of 134 echinococcosis patients, 122 patients $(110 \mathrm{CE}$ and $12 \mathrm{AE}$ ) agreed to provide blood samples (Table 3). These samples were used to evaluate diagnostic performance of diverse $\mathrm{rEgAgB}$ isoforms.

\section{Immunoreactivity profiles of $\mathrm{rEgAgBs}$ against patients' sera at different CE stages}

We retrieved representative sequences encoding EgAgB1-5, whose sequence identities ranged between $37.65-71.91 \%$ at the mRNA level (Additional file 3: Figure S2a). We expressed recombinant proteins in a prokaryotic system after fusion with GST. The GST-tag was cleaved with human rhinovirus type $143 \mathrm{C}$ protease.
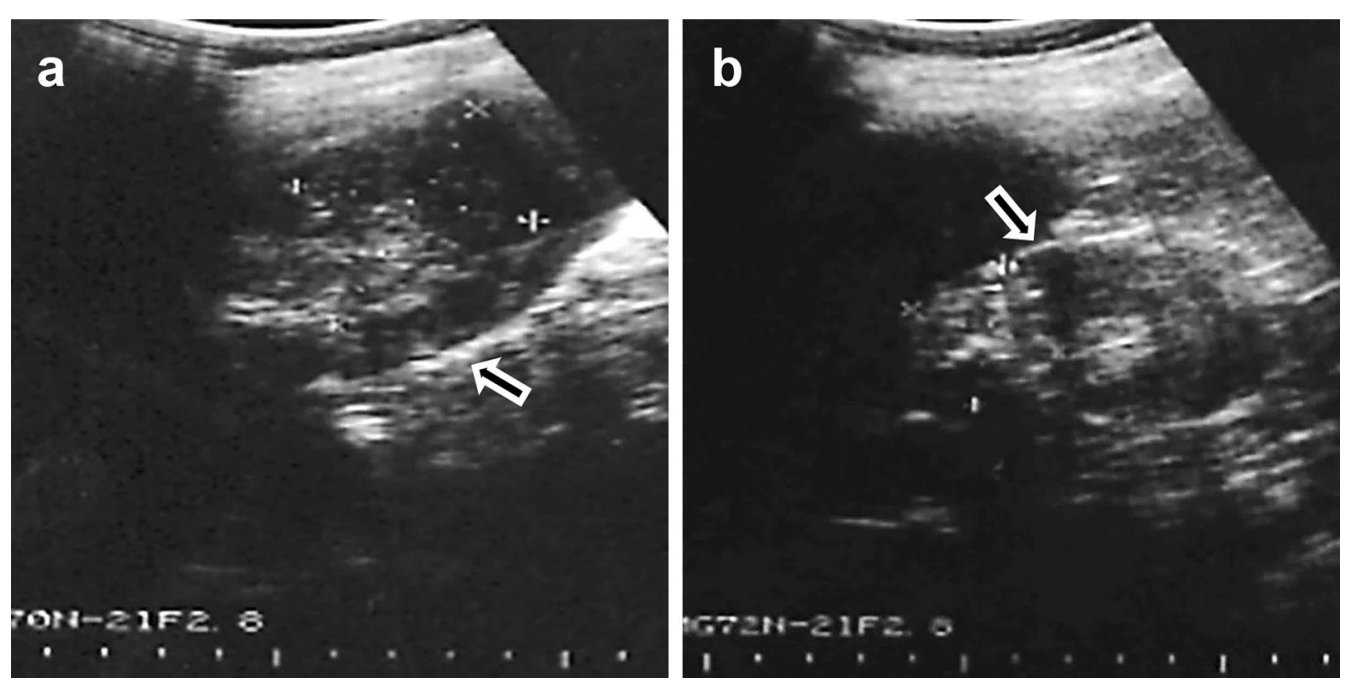

Fig. 2 Ultrasonogram of a 48-year-old herdsman who might be concomitantly infected with cystic echinococcosis (CE) and alveolar echinococcosis (AE). a Ultrasonographic (US) examination revealing a $42 \times 60 \mathrm{~mm}$ sized cystic lesion with diffuse internal calcification and hyperechoic indistinct margin (arrow) on the right hepatic lobe (CE5 stage). b Another view of the US scan showing a $74 \times 50 \mathrm{~mm}$ sized cystic mass with clusters of multiple echogenic small nodules and a calcified margin (arrow) on the right hepatic lobe (early AE stage) 
Table 3 Serodiagnostic performance of recombinant E. granulosus antigen Bs (rEgAgBs) against serum samples from different stages of cystic echinococcosis (CE) and alveolar echinococcosis (AE) cases

\begin{tabular}{|c|c|c|c|c|c|c|}
\hline & \multirow[t]{2}{*}{ No. tested } & \multicolumn{5}{|l|}{ No. positive (\%) } \\
\hline & & rEgAgB1 & rEgAgB2 & rEgAgB3 & rEgAgB4 & rEgAgB5 \\
\hline CE1 & 26 & $21(80.8)$ & 24 (92.3) & $23(88.5)$ & $16(61.5)$ & $21(80.8)$ \\
\hline CE2 & 19 & $16(84.2)$ & 18 (94.7) & $18(94.7)$ & $12(63.2)$ & 14 (73.7) \\
\hline CE3a & 10 & $9(90.0)$ & $10(100)$ & $10(100)$ & $9(90.0)$ & $9(90.0)$ \\
\hline CE3b & 20 & $20(100)$ & $20(100)$ & $20(100)$ & $16(80.0)$ & $15(75.0)$ \\
\hline CE4 & 22 & $18(81.8)$ & 20 (90.9) & 18 (81.8) & $15(68.2)$ & $20(90.9)$ \\
\hline CE5 & 13 & $9(69.2)$ & $10(76.9)$ & $10(76.9)$ & $7(53.8)$ & $9(69.2)$ \\
\hline $\mathrm{AE}$ & 12 & $6(50.0)$ & $4(33.3)$ & $4(33.3)$ & $9(75.0)$ & 10 (83.3) \\
\hline Controls & 62 & $0(0)$ & $0(0)$ & $0(0)$ & $9(14.5)$ & $17(27.4)$ \\
\hline Sensitivity & 110 & $93(84.5)$ & $102(92.7)$ & $99(90.0)$ & 75 (68.2) & $88(80.0)$ \\
\hline Specificity & 74 & $6(91.9)$ & $4(94.6)$ & $4(94.6)$ & $18(75.7)$ & $27(63.5)$ \\
\hline $\mathrm{AUC} \pm \mathrm{SE}$ & & $0.9216 \pm 0.02007$ & $0.9613 \pm 0.01283$ & $0.9517 \pm 0.01641$ & $0.8049 \pm 0.03247$ & $0.7693 \pm 0.03562$ \\
\hline $95 \% \mathrm{Cl}$ & & $0.8823-0.9610$ & $0.9362-0.9864$ & $0.9196-0.9839$ & $0.7413-0.8686$ & $0.6995-0.8391$ \\
\hline Cut-offs $^{a}$ & & 0.1625 & 0.1760 & 0.2000 & 0.1705 & 0.1190 \\
\hline PLR & & 10.43 & 17.15 & 16.65 & 2.8 & 2.19 \\
\hline NLR & & 0.17 & 0.08 & 0.11 & 0.42 & 0.31 \\
\hline PPV (\%) & & 93.9 & 96.2 & 96.1 & 80.7 & 76.5 \\
\hline NPV (\%) & & 80.0 & 89.7 & 86.4 & 61.5 & 68.1 \\
\hline Youden's index & & 0.76 & 0.87 & 0.85 & 0.44 & 0.44 \\
\hline
\end{tabular}

${ }^{\text {a }}$ Cut-off values were determined by analysis of ROC curves

Abbreviations: AE, alveolar echinococcosis; AUC, area under the ROC curve; $\mathrm{CE}$, cystic echinococcosis; Cl, confidence interval; NLR, negative likelihood ratio; NPV, negative predictive value; $r E g A g B$, recombinant $E$. granulosus antigen $\mathrm{B}$; SE, standard error; PLR, positive likelihood ratio; PPV, positive predictive value

Tricine SDS-PAGE (10\%) revealed that rEgAgB1 to rEgAgB5 migrated along with their predictive molecular weights of 7.6, 8.2, 7.9, 8.2 and $7.5 \mathrm{kDa}$, respectively. Their purity exceeded $98.5 \%$ by densitometric analyses (Additional file 3: Figure S2b).

We evaluated differential immunoreactivity of the distinct rEgAgB isoforms employing sera from patients with different $\mathrm{CE}$ stages. rEgAgB1, rEgAgB2 and rEgAgB3 showed fairly high antibody reactivity whereas rEgAgB4 and rEgAgB5 exhibited relatively weak antibody responses (Fig. 3). The optimal cut-off values determined by analyzing the ROC curves demonstrated that absorbance of 0.1625 for rEgAgB1, 0.1760 for rEgAgB2 and 0.2000 for $\mathrm{rEgAgB} 3$ distinguished the positive and negative reactions with 95\% CIs of $0.8823-0.9610$ ( $\mathrm{rEgAgB} 1)$, 0.9362-0.9864 (rEgAgB2) and 0.9196-0.9839 (rEgAgB3). These ROC curves further indicated that rEgAgB2 was the most promising biomarker, followed by rEgAgB3 and rEgAgB1 (Fig. 4). Youden's index was 0.87 for rEgAgB2, 0.85 for rEgAgB3 and 0.76 for rEgAgB1. Overall diagnostic sensitivity and specificity of rEgAgB1-3 ranged between $84.5-92.7 \%$ and 91.9-94.6\%, respectively. PLR/NLR values of rEgAgB1, 2 and 3 were 10.43/0.17, $17.15 / 0.08$ and 16.65/011, respectively. Relevant statistical variables such as sensitivity/specificity, AUC, 95\% CI,
Youden's index, PLR/NLR and PPV/NPV of individual rEgAgBs are summarized in Table 3.

Specific antibody levels between CE3a $(n=10)$ and CE3b $(n=21)$ for $\mathrm{rEgAgBs}$ were not significantly different $\left(\mathrm{rEgAgB} 1: t_{(29)}=0.3441, P=0.7333 ; \mathrm{rEgAgB} 2\right.$ : $t_{(29)}=0.4481, \quad P=0.6574 ; \quad$ rEgAgB3: $\quad t_{(29)}=0.0143$, $P=0.9887$; rEgAgB4: $t_{(29)}=0.7724, P=0.4461 ;$ rEgAgB5: $t_{(29)}=0.2995, P=0.7667$ ) (Fig. 3).

A previous study has been shown that EgAgB1 might be a promising biomarker for determining cyst viability [16]. However, when we compared the differential diagnostic performance of $\mathrm{rEgAgB} 1$ to discriminate active infections from chronic stages, rEgAgB1 did not show such a significant difference, although rEgAgB1 demonstrated lower antibody responses against sera from chronic stages of CE4 $\left(F_{(3,65)}=2.4609, P=0.0935\right)$ and CE5 $\left(F_{(3,38)}=2.2949, P=0.7464\right)$ to some extent (Fig. 3$)$.

We further assessed cross-reactivity attributed to different $\mathrm{rEgAgB}$ proteins to analyze serodiagnostic feasibility. As shown in Table 3 and Fig. 3, 50\% of serum samples from patients with AE exhibited false positive reactions against rEgAgB1. Conversely, 33.3\% of sera from $\mathrm{AE}$ cases showed cross-reactivity with $\mathrm{rEgAgB2}$ or rEgAgB3. rEgAgB4 and rEgAgB5 displayed 75 and 83.3\% cross-reactions against serum samples from $\mathrm{AE}$ patients 


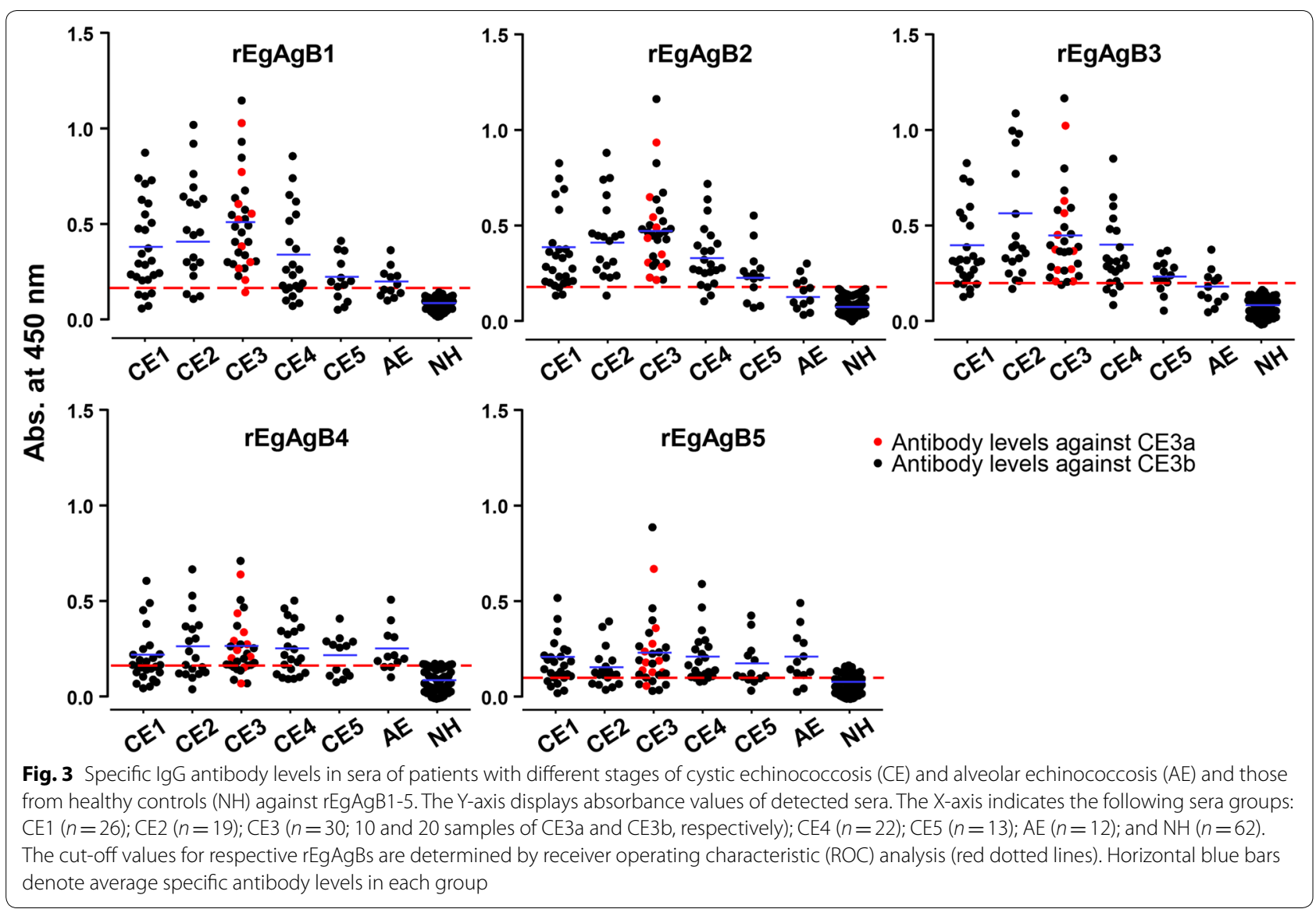

examined. Serum samples obtained from age- and sexmatched healthy donors who were the same villagers with similar occupations and dog owners showed no crossreaction against rEgAgB1, rEgAgB2 or rEgAgB3. Conversely, these sera demonstrated cross-reactions against rEgAgB4 and rEgAgB5 at rates of 14.5\% (9/62 samples) and $27.4 \%$ (17/62 sera), respectively.

\section{Discussion}

In this study, we surveyed the endemicity of echinococcoses by abdominal US examination at remote mountainous seminomadic area of southeastern Qinghai-Tibetan Plateau (Golog and Yushu Tibetan Autonomous Prefectures). We also collected serum samples to analyze specific antibody levels against diverse rEgAgBs to evaluate their diagnostic feasibility when both $\mathrm{CE}$ and $\mathrm{AE}$ are coendemic. The overall infection rate of echinococcoses reached up to $4.7 \%$ (115 CE cysts and $20 \mathrm{AE}$ masses in 134 patients) among the 2856 people examined. The majority of patients suffered from CE (4.0\%) and those suffering from AE constituted a minor fraction (0.7\%). When we assessed the diagnostic applicability of rEgAgB isoforms employing serum samples from these patients,
rEgAgB2 demonstrated the most promising potential as a CE biomarker. rEgAgB3 and rEgAgB1 also exhibited consistent diagnostic performance. Conversely, rEgAgB4 and rEgAgB5 showed low sensitivity with high cross reactions against serum samples from AE patients as well as those from healthy donors. We could not analyze immune responses of one patient who might be concomitantly infected with $\mathrm{CE}$ and $\mathrm{AE}$ due to his refusal to draw a blood sample.

Both $\mathrm{CE}$ and $\mathrm{AE}$ are known to be endemic in Qinghai Province and are associated with significant disabilityadjusted life years. However, reports on the actual prevalence rate of echinococcoses in the Qinghai-Tibetan Plateau, based on US examination, has been relatively limited [27, 28, 32]. Our survey strongly suggests that public health concerns related to echinococcoses in this area might be more serious than hitherto considered $[8,27,28,32]$. The prevalence of echinococcoses has recently been reported, ranging between 1.7-2.1\% (0.6$0.8 \%$ for $\mathrm{CE}$ and $1.1-1.3 \%$ for $\mathrm{AE}$ ) among school children in the Qinghai-Tibetan area [27, 28]. We extended our survey to adult populations to figure out general prevalence of echinococcoses in this endemic area. Our 


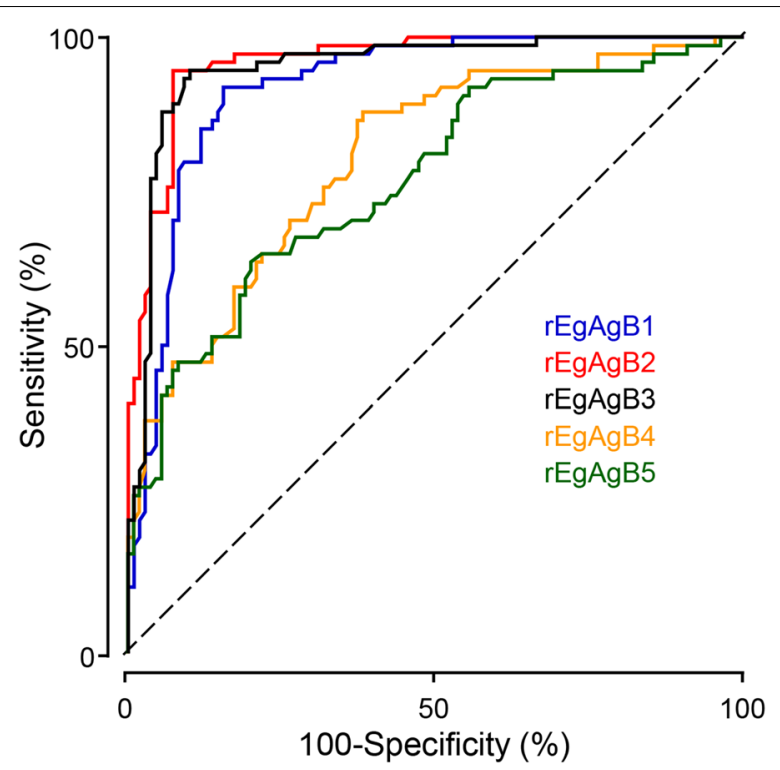

Fig. 4 Receiver operating characteristic (ROC) curves for rEgAgB1-5 assay in serum samples by ELISA. These curves represent plots of sensitivity (\%) versus 100-specificity (\%) for respective rEgAgBs. The reference line is also shown (dotted diagonal line). ROC curves are used to determine cutoff values and areas under the curve (AUC) each for rEgAgB isoforms against serum samples from patients with CE $(n=110)$

results demonstrated somewhat different epidemiological characteristics compared to previous studies [27, 28]. We observed a higher prevalence rate of echinococcoses (4.7\%) than those in earlier studies. We surmised that our study may not have detected all cases because we did not conduct lung examinations, which is the second most predilection site for CE [1]. There was no gender difference in our study, while previous studies have shown a high prevalence in female children [27, 28]. Although the number of AE patients detected was not statistically significant, patients with AE might suffer from the disease starting at earlier ages (age range: $8-48$ years; mean: 22.7 years) compared to those with $\mathrm{CE}$ (age range: 6-88 years; mean: 37.5 years) (Tables 1,2 ). CE patients were largely $(67.5 \%)$ diagnosed at active and transitional stages of CE1-3, while a considerable proportion (60\%) of $\mathrm{AE}$ patients were detected at an advanced stage (Table 2).

We observed fewer AE cases compared to those in previous studies [27, 28, 32]. This result might be attributable to the fact that we examined reasonably small numbers of school children comparted to previous studies. Moreover, patients who had already started disease treatment were excluded from statistical analyses. Alternatively, the endemicity of AE might be restricted to relatively small area, mostly confined to Golog Prefecture (Dari and Banma Counties) as reported previously [27, 28, 32].
Golog and Yushu Prefectures are seminomadic areas where lots of ungulates graze in a wide meadow. Dogs are raised to manage cattle. In the nomadic way of life, women are responsible for several households during which they might be exposed to dogs and their feces contaminated with CE eggs. They might become infected with CE. Men might be exposed to E. granulosus eggs while grazing cattle with their shepherd dogs in the steppe [32, 33]. These environmental, ecological and human geographical characteristics might have contributed to the active transmission of CE in these areas.

It is interesting to note that the transmission mode of $\mathrm{AE}$ in the Qinghai-Tibetan Plateau seems to be different from that in European countries. In European communities, the adult population might be more likely to contract $\mathrm{AE}$ while gardening with bare hands, hunting in the forest, or picking mushrooms in grasslands associated with fox habitats [34-36]. Conversely, AE had a high infection rate in the young age group in the QinghaiTibetan Plateau (Table 2). This epidemiological situation corroborated well with previous studies [27, 28]. Most children might be infected with AE by dirt-eating habits during playing in the steppe and/or helping parents in daily activities such as taking care of livestock and household chores. They usually gather dry animal excrement, which might be mixed with that of dogs, as major sources of fuel materials. These traditional social behaviors might have played major roles in the transmission of $\mathrm{AE}$ among children in these areas $[37,38]$.

Our previous analysis involving the immunoproteome profile of native EgAgB proteins has demonstrated that EgAgB1 comprises the most abundant fraction in both CE1 and CE2 HF and that EgAgB1 is the most sensitive antigen [15]. Neither EgAgB2 nor EgAgB3 exhibited such sensitive responses but showed positive reactions mainly to sera from patients with CE2 and CE3 stages, during which host immune responses might be highly active [15]. Other serodiagnostic studies using synthetic EgAgBs have also indicated the high antibody capturing activity of EgAgB1 $[16,25]$. However, when we analyzed differential antibody reactivity of each recombinant protein, the order of diagnostic performance from high to low was: $\mathrm{rEgAgB} 2>\mathrm{rEg}$ AgB3 > rEgAgB1 > rEgAgB4 > rEgAgB5 (Figs. 3, 4). Furthermore, rEgAgB2 and rEgAgB3 exhibited less cross-reaction against sera from patients with AE than rEgAgB1 (Table 3). Previous studies with $\mathrm{rEgAgB} 2$ have also revealed a reliable diagnostic applicability [14, 22, 23]. Moreover, a multimeric form of EgAgB2 protein (B2t) manipulated by tandem ligation of a single nucleotide sequence of mature EgAgB2 domain has shown to significantly enhance diagnostic feasibility [14]. Although the antigenic reactivity of native EgAgB2 was not satisfactory probably due to its low expression levels in native HF $[15,39]$, the EgAgB2 molecule 
might have epitope(s) specific to CE. The generation of a chimeric protein through the combination of domain(s) harboring specific epitopes might have an advantage not only for improving $\mathrm{CE}$ serodiagnosis, but also for differential diagnosis between $\mathrm{CE}$ and $\mathrm{AE}$. The use of relevant chimeric antigen significantly improved the serodiagnostic reliability [40].

Synthetic EgAgB1 has been reported to be a promising biomarker for determining cyst viability, thus providing a clue to differentially diagnose active stage $\mathrm{CE}$ from chronic cases [16]. However, rEgAgB1 did not show such a significant difference, although rEgAgB1 demonstrated lower antibody responses against sera from chronic stages of CE4 and CE5 (Fig. 3). This conflicting observation suggests that antibody reactivity of rEgAgB1 might be somewhat different depending on the serum sample tested. Antibody responses mounted to a certain antigen(s) might reflect several important factors, including immunological status of the infected individual and biological reactivity of parasite molecules during host-parasite interactions. We assumed that serological responsiveness might be more importantly determined by host factors than by parasite factors [41]. To precisely address the differential antibody reactivity mounted to each $\mathrm{EgAgB}$ isoform, host factors involved in serological responsiveness should be carefully considered in future investigations.

\section{Conclusions}

Our results indicate that public health concerns related to echinococcoses in the Qinghai-Tibetan area seem to be more serious than previously considered. Abdominal US should still be the diagnosis of choice. Detection of specific antibody levels against rEgAgB2, rEgAgB3 and/ or rEgAgB1 might provide supportive data for the diagnosis of CE. However, in areas where both CE and AE are co-endemic, serological tests should be carefully interpreted for differential diagnosis. The transmission mode of $\mathrm{AE}$ in the Qinghai-Tibetan Plateau might be different from that in other communities. Further studies are urgently needed to assess transmission potentials of echinococcoses in this area. Interventions for disease control are also warranted to ensure patients' health and reduce social burdens.

\section{Additional files}

Additional file 1: Figure S1. Representative ultrasonographic findings of cystic echinococcosis (CE) and alveolar echinococcosis (AE) patients in this study. a Imaging scans of CE patients. CE1 stage shows well-defined cyst wall with floating and sedimenting echoes (snow-flake sign). Ultrasonograms of some patients also show double-line sign. CE2 stage demonstrates multiple daughter cysts within a unilocular large cyst. CE3a stage displays detachment of the pericystium and CE3b exhibits multiloculated daughter cysts with solid matrix. CE4 typically shows canalicular structure (ball of wool appearance). CE5 stage reveals highly degenerative cyst with calcified wall. $\mathbf{b}$ Ultrasonographic findings of AE cases. Sonogram of early AE case demonstrates clusters of multiple echogenic small nodules (hailstorm patterns) with indistinct margin with/without punctate calcifications. Advanced cases reveal a large central hypoechogenicity combined with peripheral hyperechoic indistinct and irregular border.

Additional file 2: Table S1. Gene-specific oligonucleotide primers used. Additional file 3: Figure S2. a Comparison of homology values among EgAgB1-5 at mRNA level. b Expression and purification of recombinant EgAgBs (rEgAgBs). rEgAgBs fused with GST were induced with 1 mM IPTG and purified using GSH-Sepharose 4B. GST-tags were removed by PreScission protease. The homogeneity of purified proteins was analyzed by Tricine SDS-PAGE (10\%) with CBB staining. Lanes 1-5 represent respective recombinant proteins. $M_{r}$, molecular weight in $\mathrm{kDa}$.

\section{Abbreviations}

AE: alveolar echinococcosis; AUC: area under the ROC curve; CE: cystic echinococcosis; Cl: confidence interval; EgAgB: E. granulosus antigen B; EgAg5: E. granulosus antigen 5; ELISA: enzyme-linked immunosorbent assay; EmAgB: E. multilocularis antigen B; HF: hydatid fluid; NLR: negative likelihood ratio; NPV: negative predictive value; PBS/T: phosphate buffered saline containing 0.05\% Tween 20; PLR: positive likelihood ratio; PPV: positive predictive value; rEgAgB: recombinant EgAgB; ROC: receiver operating characteristic; US: ultrasonography.

\section{Acknowledgements}

We thank all local health workers of the Qinghai Province Institute for Endemic Diseases Prevention and Control, China, who helped us during the survey of patients.

\section{Authors' contributions}

Experimental conception and design: $Y K, X \mathrm{XH}, \mathrm{JGK}$ and HW. Ultrasonographic examination: $X H, H C, X M$ and HW. Sample collections: XH, HW, HC and XM. Cloning and expression: JGK, CSA, DHD. ELISA: JGK, CSA and DHD. Data analysis: YK, JGK, CSA and IK. Paper writing: YK, JGK, CSA and IK. All authors read and approved the final manuscript.

\section{Funding}

This work was supported by the Qinghai Province International Science and Technology Cooperation Project, China (2019-HZ-803) and also by the National Research Foundation, Korea (NRF-2016R1A6A3A1 1931725).

\section{Availability of data and materials}

The data supporting the conclusions are included within the article and its additional files. The raw data used or analyzed during the study are available from the corresponding author upon reasonable request.

\section{Ethics approval and consent to participate}

The use of serum samples was approved by the Institutional Review Committee of Qinghai Province Institute for Endemic Diseases Prevention and Control, China (Protocol no. 2013-7-22). Written informed consent was obtained from each patient and participant. In the case of illiterate patients, consent was obtained by verbal notification. These sera were anonymized in this study.

\section{Consent for publication}

Not applicable.

\section{Competing interests}

The authors declare that they have no competing interests.

\section{Author details}

${ }^{1}$ Qinghai Provincial People's Hospital, Xining 810007, China. ${ }^{2}$ Qinghai Province Institute for Endemic Diseases Prevention and Control, Qinghai Centers for Disease Prevention and Control, Xining 811602, China. ${ }^{3}$ Department of Molecular Parasitology, Samsung Medical Center, Sungkyunkwan University School of Medicine, Suwon 16419, Korea. ${ }^{4}$ Endemic Disease Administration 
Office, Qinghai Province Health and Family Planning Commission, Xining 811602, China. ${ }^{5}$ Department of Molecular Biology and Biochemistry, Kyung Hee University College of Medicine, Seoul 02447, Korea.

Received: 6 December 2018 Accepted: 17 June 2019 Published online: 26 June 2019

\section{References}

1. Eckert J, Deplazes P. Biological, epidemiological, and clinical aspects of echinococcosis, a zoonosis of increasing concern. Clin Microbiol Rev. 2004;17:107-35.

2. Budke CM, Deplazes P, Torgerson PR. Global socioeconomic impact of cystic echinococcosis. Emerg Infect Dis. 2006;12:296-303.

3. Craig PS, McManus DP, Lightowlers MW, Chabalgoity JA, Garcia HH, Gavidia CM, et al. Prevention and control of cystic echinococcosis. Lancet Infect Dis. 2007;7:385-94.

4. Piarroux M, Piarroux R, Giorgi R, Knapp J, Bardonnet K, Sudre B, et al. Clinical features and evolution of alveolar echinococcosis in France from 1982 to 2007: results of a survey in 387 patients. J Hepatol. 2011;55:1025-33.

5. Schmidberger J, Kratzer W, Stark K, Grüner B, Echinococcosis Working Group. Alveolar echinococcosis in Germany, 1992-2016. An update based on the newly established national AE database. Infection. 2018:46:197-206.

6. Spicher M, Naguleswaran A, Ortega-Mora LM, Müller J, Gottstein B, Hemphill A. In vitro and in vivo effects of 2-methoxyestradiol, either alone or combined with albendazole, against Echinococcus metacestodes. Exp Parasitol. 2008;119:475-82.

7. Zhang T, Zhao W, Yang D, Piao D, Huang S, Mi Y, et al. Human cystic echinococcosis in Heilongjiang Province, China: a retrospective study. BMC Gastroenterol. 2015;15:29.

8. Zhang W, Zhang Z, Wu W, Shi B, Li J, Zhou X, et al. Epidemiology and control of echinococcosis in central Asia, with particular reference to the People's Republic of China. Acta Trop. 2015;141:235-43.

9. Bulakçi M, Kartal MG, Yılmaz S, Yılmaz E, Yılmaz R, Şahin D, et al. Multimodality imaging in diagnosis and management of alveolar echinococcosis: an update. Diagn Interv Radiol. 2016;22:247-56.

10. Tamarozzi F, Hou A, Morales ML, Giordani MT, Vilca F, Mozo K, et al. Prevalence and risk factors for human cystic echinococcosis in the Cusco region of the Peruvian highlands diagnosed using focused abdominal ultrasound. Am J Trop Med Hyg. 2017;96:1472-7.

11. Solomon N, Zeyhle E, Subramanian K, Fields PJ, Romig T, Kern P, et al. Cystic echinococcosis in Turkana, Kenya: 30 years of imaging in an endemic region. Acta Trop. 2018;178:182-9.

12. Mirabile E, Solomon N, Fields PJ, Macpherson CNL. Progress towards international adoption of the World Health Organization ultrasound classification of cystic echinococcosis. Acta Trop. 2019;189:6-9.

13. Siles-Lucas M, Casulli A, Conraths FJ, Müller N. Laboratory diagnosis of Echinococcus spp. in human patients and infected animals. Adv Parasitol. 2017;96:159-257.

14. Hernández-González A, Santivañez S, García HH, Rodríguez S, Muñoz S, Ramos $\mathrm{G}$, et al. Improved serodiagnosis of cystic echinococcosis using the new recombinant 2B2t antigen. PLoS Negl Trop Dis. 2012;6:e1714.

15. Ahn CS, Han X, Bae YA, Ma X, Kim JT, Cai H, et al. Alteration of immunoproteome profile of Echinococcus granulosus hydatid fluid with progression of cystic echinococcosis. Parasit Vectors. 2015;8:10.

16. Pagnozzi D, Tamarozzi F, Roggio AM, Tedde V, Addis MF, Pisanu S, et al. Structural and immunodiagnostic characterization of synthetic antigen B subunits from Echinococcus granulosus and their evaluation as target antigens for cyst viability assessment. Clin Infect Dis. 2018;66:1342-51.

17. Lorenzo C, Last JA, González-Sapienza GG. The immunogenicity of Echinococcus granulosus antigen 5 is determined by its post-translational modifications. Parasitology. 2005;131:669-77.

18. Ahn CS, Bae YA, Kim SH, Kim JG, Yu JR, Yang HJ, et al. Spatiotemporal expression patterns and antibody reactivity of Taeniidae endophilin B1.J Clin Microbiol. 2016;54:2553-62.
19. Ahn CS, Kim JG, Han X, Bae YA, Park WJ, Kang I, et al. Biochemical characterization of Echinococcus multilocularis antigen $\mathrm{B} 3$ reveals insight into adaptation and maintenance of parasitic homeostasis at the host-parasite interface. J Proteome Res. 2017;16:806-23.

20. Ahn CS, Cai H, Kim JG, Han X, Ma X, Bae YA, et al. An Echinococcus multilocularis antigen $\mathrm{B} 3$ proteoform that shows specific antibody responses to active-stage alveolar echinococcosis. J Clin Microbiol. 2015;53:3310-7.

21. Zhang W, Li J, Jones MK, Zhang Z, Zhao L, Blair D, McManus DP. The Echinococcus granulosus antigen $B$ gene family comprises at least 10 unique genes in five subclasses which are differentially expressed. PLoS Negl Trop Dis. 2010;4:e784.

22. Virginio VG, Hernández A, Rott MB, Monteiro KM, Zandonai AF, Nieto A, et al. A set of recombinant antigens from Echinococcus granulosus with potential for use in the immunodiagnosis of human cystic hydatid disease. Clin Exp Immunol. 2003;132:309-15.

23. Jiang $L$, Zhang $Y G$, Liu MX, Feng $Z$. Analysis on the reactivity of five subunits of antigen $B$ family in serodiagnosis of echinococcosis. Exp Parasitol. 2012;131:85-91.

24. Rott MB, Fernández V, Farias S, Ceni J, Ferreira HB, Haag KL, et al. Comparative analysis of two different subunits of antigen $B$ from Echinococcus granulosus: gene sequence, expression in Escherichia coli and serological evaluation. Acta Trop. 2000;75:331-40

25. González-Sapienza G, Lorenzo C, Nieto A. Improved immunodiagnosis of cystic hydatid disease by using a synthetic peptide with higher diagnostic value than that of its parent protein, Echinococcus granulosus antigen B. J Clin Microbiol. 2000;38:3979-83.

26. de la Rue ML, Yamano K, Almeida CE, lesbich MP, Fernandes CD, Goto A, et al. Serological reactivity of patients with Echinococcus infections ( $E$. granulosus, E. vogeli, and E. multilocularis) against three antigen B subunits. Parasitol Res. 2010;106:741-5.

27. Cai H, Guan Y, Ma X, Wang L, Wang H, Su G, et al. Epidemiology of echinococcosis among schoolchildren in Golog Tibetan Autonomous Prefecture, Qinghai, China. Am JTrop Med Hyg. 2017;96:674-9.

28. Han XM, Cai QG, Wang W, Wang H, Zhang Q, Wang YS. Childhood suffering: hyper endemic echinococcosis in Qinghai-Tibetan primary school students, China. Infect Dis Poverty. 2018;7:71.

29. Brunetti E, Kern P, Vuitton DA. Writing panel for the WHO-IWGE. Expert consensus for the diagnosis and treatment of cystic and alveolar echinococcosis in humans. Acta Trop. 2010;114:1-16.

30. Kern P, Wen H, Sato N, Vuitton DA, Gruener B, Shao Y, et al. WHO classification of alveolar echinococcosis: principles and application. Parasitol Int. 2006;55:S283-7.

31. Grüner B, Kern P, Mayer B, Gräter T, Hillenbrand A, Barth TEF, et al. Comprehensive diagnosis and treatment of alveolar echinococcosis: a single-center, long-term observational study of 312 patients in Germany. GMS Infect Dis. 2017;5:Doc01.

32. Yu SH, Wang H, Wu XH, Ma X, Liu PY, Liu YF, et al. Cystic and alveolar echinococcosis: an epidemiological survey in a Tibetan population in southeast Qinghai, China. Jpn J Infect Dis. 2008;61:242-6.

33. Feng X, Qi X, Yang L, Duan X, Fang B, Gongsang Q, et al. Human cystic and alveolar echinococcosis in the Tibet Autonomous Region (TAR), China. J Helminthol. 2015;89:671-9.

34. Kern P, Ammon A, Kron M, Sinn G, Sander S, Petersen LR, et al. Risk factor for alveolar echinococcosis in humans. Emerg Infect Dis. 2004;10:2088-93.

35. Antolova D, Miterpakova M, Radoňak J, Hudačkova D, Szilagyiova M, Začek M. Alveolar echinococcosis in a highly endemic area of Northern Slovakia between 2000 and 2013. Eurosurveillance. 2014. https://doi. org/10.2807/1560-7917.ES2014.19.34.20882.

36. Gottstein B, Stojkovic M, Vuitton DA, Millon L, Marcinkute A, Deplazes P. Threat of alveolar echinococcosis to public health—a challenge for Europe. Trends Parasitol. 2013;31:407-12.

37. Schantz PM, Wang H, Qiu J, Liu FJ, Saito E, Emshoff A, et al. Echinococcosis on the Tibetan Plateau: prevalence and risk factors for cystic and alveolar echinococcosis in Tibetan populations in Qinghai Province, China. Parasitology. 2003;127:S109-20. 
38. Craig PS. Epidemiology of human alveolar echinococcosis in China. Parasitol Int. 2006;55:S221-5.

39. Ahn CS, Kim JG, Han X, Kang I, Kong Y. Comparison of Echinococcus multilocularis and Echinococcus granulosus hydatid fluid proteome provides molecular strategies for specialized host-parasite interactions. Oncotarget. 2017;8:97009-24.

40. Bae YA, Jeong YT, Chung JY, Kim SH, Mahanta J, Feng Z, et al. A recombinant chimeric antigen toward a standardized serodiagnosis of Taenia solium neurocysticercosis. Proteom Clin Appl. 2008:2:1596-610.
41. Chung JY, Bahk YY, Huh S, Kang SY, Kong Y, Cho SY. A recombinant 10-kDa protein of Taenia solium metacestodes specific to active neurocysticercosis. J Infect Dis. 1999:180:1307-15.

\section{Publisher's Note}

Springer Nature remains neutral with regard to jurisdictional claims in published maps and institutional affiliations.
Ready to submit your research? Choose BMC and benefit from:

- fast, convenient online submission

- thorough peer review by experienced researchers in your field

- rapid publication on acceptance

- support for research data, including large and complex data types

- gold Open Access which fosters wider collaboration and increased citations

- maximum visibility for your research: over $100 \mathrm{M}$ website views per year

At BMC, research is always in progress.

Learn more biomedcentral.com/submissions 